\title{
Plastic films for polytunnels can prolong the effective residual life of cypermethrin to over 6 months
}

Article

Accepted Version

van Emden, H. F. and Hadley, P. (2011) Plastic films for polytunnels can prolong the effective residual life of cypermethrin to over 6 months. Journal of Horticultural Science \& Biotechnology, 86 (2). pp. 196-200. ISSN 14620316 doi: https://doi.org/10.1080/14620316.2011.11512747 Available at https://centaur.reading.ac.uk/19592/

It is advisable to refer to the publisher's version if you intend to cite from the work. See Guidance on citing.

To link to this article DOI: http://dx.doi.org/10.1080/14620316.2011.11512747

Publisher: Taylor \& Francis

All outputs in CentAUR are protected by Intellectual Property Rights law, including copyright law. Copyright and IPR is retained by the creators or other copyright holders. Terms and conditions for use of this material are defined in the End User Agreement.

www.reading.ac.uk/centaur 
Central Archive at the University of Reading

Reading's research outputs online 
Plastic films for polytunnels can prolong the effective residual life of cypermethrin to over six months

\section{By HELMUT F. VAN EMDEN* and PAUL HADLEY}

Centre for Horticulture and Landscape, School of Biological Sciences, University of Reading, Whiteknights, Reading, Berkshire, RG6 6AS, UK

(e-mail: h.f.vanemden@reading.ac.uk)

Running head: Cypermethrin residual activity in polytunnels

*Author for correspondence 


\section{SUMMARY}

The synthetic pyrethroid insecticide is degraded almost entirely by ultraviolet (UV) catalysed oxidation. A bioassay with the beetle Tribolium confusum duVal caged on bandage soaked in $0.04 \%$ a.i. cypermethrin showed large differences in residual life under three plastics available for polytunnels. A UV film transmitting $70 \%$ of UVB and $80 \%$ of UVA killed all beetles for eight weeks compared with only three weeks in a clear plastic envelope treatment. A UV absorbing film reduced transmission of UVB and UVA to 14 and $50 \%$ respectively, and gave complete kill for 17 weeks. Reducing transmission of UVB to virtually zero and UVA to only $3 \%$ with a UV opaque film prolonged the effective residue to 26 weeks, and some beetles were still killed for 11 more weeks. Even thereafter, the beetles in the UV opaque treatment were still affected by the pesticide, and only showed near normal mobility 23 months after pesticide application. These results have implications for recommending intervals between cypermethrin treatment and harvest or introduction of insect biological control agents where UV opaque films are used in horticulture. 
I the early part of the 20th century, one of the most important insecticides widely used in agriculture and horticulture was natural pyrethrum, extracted from the flowers of several Old World species of Chrysanthemum (e.g. $C$. cinerariifolium Treviranus and $C$. coccineum Willdenow). The problem with this insecticide was its extremely short residual life, for it lasted a fraction of an hour and it was really necessary to contact the target insects with spray at the time of application. The short residual life stemmed from the rapid oxidation of the pyrethrum in daylight, sincethe oxidation requires catalysis from ultraviolet light (UV) - a degradation process known as "photochemical oxidation" (Fahmy et al., 1978; Cole et al., 1982).

However, natural pyrethrum had the major advantage of low mammalian toxicity, and so the 20th century saw continuing efforts over many years to imbue pyrethroids with photostability. Although partial success came with the synthesis in the USA of allethrin as early as the 1940s (Schechter et al., 1949), the real breakthrough was achieved in the early 1970s by a group at Rothamsted Experimental Station (now Rothamsted Research) (Elliott et al., 1973). As a result the synthetic pyrethroids were born, and a number of active ingredients were made available to the agrochemical industry for further development. Cypermethrin, the pyrethroid used in this study, was acquired by what is now Syngenta.

In recent years, a range of new plastics has been developed for cladding polytunnels in horticulture. One motivation for reducing UV transmission by such plastics was the discovery that such plastics controlled a number of plant diseases (Sasaki et al., 1985) including grey mould caused by Botrytis spp., a common problem in protected crops. More recently (Doukas, 2002; Doukas and Payne, 2007)) it has been shown that glasshouse whitefly (Trialeurodes vaporariorum (Westwood)) was less flight-active under UV opaque plastic, and that in choice tests both the whitefly and its parasitoid (Encarsia formosa Gahan) preferred to collect under UV transparent plastic film. 
This study involved three plastics, all produced by British Polythene Industries plc (BPI), UK. One of these new claddings was a UV opaque film, which blocks UV up to $380 \mathrm{~nm}$ (all UVB and most of UVA), and so might be expected to prolong the residual life of pyrethroids. Another, UV transmitting, is the opposite, and transmits the full UV range. By comparison, the standard plastic film (UV low film) gives low transmission of UV light up to $330 \mathrm{~nm}$, and reduced transmission compared with the UV transmitting filmin the range $330-380 \mathrm{~nm}$. All three films contained the infrared-reducing and light-diffusing components of Luminance THB (BPI plc). To the human eye, there was no discernible difference between the light transmitted by these three films. Horticultural research at Reading University had recently set up some experiments on the effect on the growth and yield of soft fruit of these films, and so an experimental set-up was already available for testing the residual life of a pyrethroid insecticide.

A preliminary trial in 2007 with Brussels sprout plants (Brassica oleracea L. v. capitata) and caterpillars of the large cabbage white butterfly (Pieris brassicae L.) showed that even a young leaf treated with $0.05 \%$ a.i. cypermethrin still had a toxic residue when it senesced and abscised from a plant kept under standard UV low film. It was therefore necessary to develop an insect mortality bioassay which did not involve plants.

\section{MATERIALS AND METHODS}

The polytunnels and cladding treatments

The experiment was performed in a seven span, 'spanish' open-ended polytunnel. Each span measured $75 \mathrm{~m}$ in length and $7.5 \mathrm{~m}$ wide. In the early winter of 2007-2008, the plastic cladding on alternate tunnels had been removed, leaving the three tunnels with cladding treatments separated by an open tunnel-wide strip where the plastic film had been removed. The cladding of these remaining tunnels was in three $25 \mathrm{~m}$ sections stitched 
together; each section was of different polythene with the three treatments allocated to sections within tunnels as shown in Figure 1.

The three cladding treatments (there was also a treatment $\mathrm{A}$ in the open outside the tunnels, see later) were B, UV transmitting; C, the standard (UV low) film; D, UV opaque film. The claddings terminated $1 \mathrm{~m}$ above soil level, leaving the lower part of the sides of the tunnels open.

At the start of December 2008, the cladding of the tunnels had to be removed to avoid storm damage, yet the experiment was still in progress. The experiment was therefore miniaturised (see below) and removed from the tunnels.

The spectral transmissions of the three claddings were measured with a Benthams Spectroradiometer (M 300 EA monochromator). As the clear envelope of treatment A and horticultural glass do not diffuse the transmitted light, readings from a standard spectrophotometer were considered reliable.

\section{Pyrethroid-treated surfaces}

Preliminary tests showed that kill of the insects to be used in the bioassay (adults of the confused flour beetle, Tribolium confusum duVal) required a relatively high concentration of cypermethrin. From a $10 \%$ EC formulation of cypermethrin, a $0.04 \%$ a.i. solution was prepared.

Cotton bandage $5 \mathrm{~cm}$ in width was cut into strips $18 \mathrm{~cm}$ long. These were immersed fully in the cypermethrin solution and then hung on a line to dry. All this was done in one of the UV opaque film tunnel sections. The strips were then laid on a piece of hardboard $12.5 \times 5.4 \mathrm{~cm}$. The ends of the bandage strips were folded over the ends of the hardboards and the bandage secured with two small bulldog clips. For untreated strips, as it was not possible to obtain the cypermethrin formulation without the active ingredient, instead 
strips were immersed in water to which $1 \mathrm{ml} \mathrm{l}^{-1}$ of the surfactant Tween 20 had been added.

Each section in the tunnels had two parallel rails $23 \mathrm{~cm}$ apart and set on posts $1 \mathrm{~m}$ high. These rails had supported bags of compost for strawberries which had previously occupied the sections. A seed tray $32 \times 20 \mathrm{~cm}$ was placed upside down on the rails near the centre of the section and wired onto them. Two hardboards with treated bandage were placed on each seed tray; the two boards in each section were identified with one of the small bulldog clips being marked either with red or blue tape (Figure 2). Additionally a seed tray was wired onto a wooden pallet in three of the open sections between the panels (Figure1) to add a fourth treatment (A), i.e. the absence of any polythene cladding. Each seed tray in the experiment was designated as a 'station'. A hardboard with untreated strips of bandage (see earlier) was included at each of the four stations representing the four treatments of replicate 1 (Figure 1). To protect the hardboards outside the filmcovered tunnels from rain, they were enclosed in a thin clear A4 punched pocket.

When the time came to miniaturise the experiment (see earlier), the hardboards from stations still involved in the experiment were placed into seed trays screwed onto a single wooden pallet in the open, and each tray had a lid of the appropriate plastic film spanned across a loosely fitting wooden frame.

\section{The bioassay}

Since the order in which the cypermethrin residues would degrade in the four treatments could be predicted, the bioassay began with only the three ' $A$ ' stations outside the tunnels.

Bioassays were conducted weekly, usually set up on Mondays. In order to reduce the percentage of time that hardboards were removed from the tunnels for bioassay, the 'red' and 'blue' series of boards at each station were used alternately. Additionally, the 
untreated bandage from the station with the greatest UV- blocking involved in the bioassay was usually included in order to check that no changes had occurred to the bandage strips that might cause mortality of the beetles. Indeed, complete survival of beetles on these controls continued for the duration of the experiment.

The hardboards required for the bioassay were collected from the tunnels and brought into the laboratory where $T$. confusum was reared in a glass jar containing some wholemeal flour and kept an incubator running at $23 \pm 1.5^{\circ} \mathrm{C}$. For each hardboard, two beetles were taken from the culture and placed into a plastic lid $2.5 \mathrm{~cm}$ in diameter. The bandage side of the hardboard was placed over the open side of the lid and both inverted. The lid was then secured with 2 bent hair-curl clips (Figure 2) and the hardboard reinverted to bring the beetles in contact with the bandage.

Mortality of the beetles was assessed after $48 \mathrm{~h}$. The beetles were considered still alive if all three pairs of legs moved in a coordinated way so that the beetles were capable of locomotion. Those incapable of movement other than the twitching of legs were classified as "dead". The hardboards were then returned to the appropriate station.

As soon as at least one of the beetles on a hardboard was still alive when the bioassay was assessed, that station was no longer included in later bioassays. When this first occurred in a particular treatment, the three stations of the next treatment in order of UVblocking were included in the next bioassay. When the beetles in the final treatment (D) had live individuals is all replicates, it was noted that they were still inactive compared with the beetles on the relevant untreated bandage. The bioassay was therefore continued, but now four beetles were caged per hardboard. The time taken for individual living beetles to move outside the caged area on the bandage was measured from the time the cage was removed under the light from a desk lamp. Observation was terminated after 2 $\min$. 


\section{RESULTS}

The spectroradiometer and spectrophotometer results (Table I and Figure 3) showed that the A4 pocket (A) transmitted $90 \%$ of the light in the visible spectrum $(401-700 \mathrm{~nm})$ but there was a $15-20 \%$ reduction of UV transmission The three plastics all reduced transmission in the visible spectrum $(401-700 \mathrm{~nm})$ to about $85 \%$, but with clear differences in transmission of UV. The UV transmitting film (B) allowed the transmission of nearly $70 \%$ in the UVB range, in contrast with only some $14 \%$ for the UV low (C) and virtually none for the UV opaque film. The three films showed somewhat greater transmission in the UVA range: approximately $80 \%$ (UV transmitting), 50\% (UV low) and a still very low value of only $3.1 \%$ for the UV opaque film.

The results of the bioassay were straightforward (Figure 4). Effective residual life of cypermethrin increased progressively as UV-blocking of the films increased, with clear separation by several weeks between the treatments. As it was impossible (see earlier) for blocking UV transmission to shorten the residual life of a synthetic pyrethroid, one way statistical comparisons are appropriate.

Outside the tunnels (treatment A in the clear A4 pockets) the first live beetle were found in replicates 1 and 2 after 4 weeks and 1 week later live beetles were found in the remaining replicate 1 (mean $=4.3$ weeks).

Under the UV transmitting film (treatment B), the cypermethrin residue was effective for more than twice as long as outside the tunnels. All beetles were killed for 8 weeks and the first live beetles were not found (in replicate 2) till week 9. As in treatment A, the remaining 2 replicates had live beetles 1 week later (week 10). The mean of 9.7 weeks was outside the $P=0.005$ confidence limits for the mean outside the tunnels.

Cypermethrin residues in treatment C, i.e. under the standard film (UV low), remained effective for much longer, again a doubling in time (mean $=19.3$ weeks, considerably 
outside the $P=0.001$ confidence limits for the mean under the UV transmitting film. The first live beetles (in replicates 1 and 3 were not found until week 18, with live beetles in found in week 20 in the last replicate (2).

Under the UV opaque film, beetles were killed for 9 weeks longer than in treatment B (a live beetle was found in replicate 2 in week 27), over half a year since the cypermethrin had been applied. In week 32 such a beetle occurred in replicate 3 and it was only in week 38 that beetles were alive in replicate 1 (mean $=32.3$ weeks, outside the $P=0.025$ confidence limits for the UV low film).

Any criterion for mortality in bioassays is only relative between treatments. In these bioassays, that beetles could walk (the criterion for "alive") did not mean they were unaffected by the insecticide. Although beetles were alive in all UV opaque film replicates by week 38, they were still clearly affected by the cypermethrin for much longer. For many weeks after week 38 , all beetles on the untreated bandage quickly left the caged area once light was shone on them, whereas no beetles on treated bandage from the UV opaque treatment achieved this until after a year had passed since cypermethrin application. Figure 5 compares the mean time taken by beetles on the untreated and treated bandage under this film from month 12 onwards. The reduction in activity with cypermethrin treatment was considerable until 22 months after application. Although the reduction was still statistically significant one month later, most beetles in the UV opaque treatment were by then leaving the caged area within 20 seconds, and after a further week (i.e. 24 months after application, when the experiment was terminated) there was no significant reduction in activity in that treatment.

\section{DISCUSSION}

It is clear that blocking transmission of UV greatly extended the effective residual life of cypermethrin to six months in the most extreme treatment (UV opaque). This is because 
photochemical oxidation is the main process by which synthetic pyrethroids are degraded (Casida, 1980). There is some evidence in our results that UVB is more important for degradation than UVA, since this was more blocked by the plastics than UVB. Moreover, UVB transmission was identical for the clear plastic pocket and the UV transmitting film, yet cypermethrin remained effective under the latter for more than twice as long.

The other main ways in which insecticide residues are depleted (van Emden and Service, 2004) are volatility, which on plant surfaces is increased by the passage of transpiration water through the insecticide film, rainwash, enzymatic degradation by microflora on leaves and the soil, and flaking/abrasion of the solid residue. Using cypermethrin-treated bandage strips on hardboard plates eliminated effects on plant surfaces, though the very low volatility of cypermethrin would probably make such losses by evaporation and transpiration negligible even in a plant-based bioassay. The polytunnels would protect the residues from rain and to a large extent abrasion of the solid residue would be small with the reduced air-movement within the tunnels. Furthermore, cypermethrin is stable to hydrolysis at normal environmental conditions and with water below pH (Jones, 1995). Thus our bioassay probably gave results that would not be misleading for residues on seasonal crops grown under protected cultivation. This is confirmed by the three-week effective residue outside the polytunnels and the preliminary experiments with plants (see earlier), where the cypermethrin residues under standard polytunnel film outlasted the lifespan of a Brussels sprout leaf treated when young.

For the much more volatile organophosphate insecticides, photochemical oxidation represents a smaller but still major cause of residue loss (Rammell and Bentley, 1990). It is therefore likely that the extension of residue life under polythene claddings would also apply, though to a lesser extent, to organophosphates (see later). Amano et al. (2002) have already shown that, under a normal vinyl film, dichlorvos residues in spinach degraded completely after three days, but only after six days under a UV opaque film. 
Complete degradation of fenitrothion took six and 95 days respectively under the same two films.

The experiment continued much longer than expected, and so extended across the winter months. This may have prolonged the residue life in the two more extreme UVblocking treatments as the days became shorter, more overcast and with the sun increasingly further away. Of course temperature also decreased with winter, but this is unlikely to have been of importance with an insecticide of such low volatility. The time between beetles surviving in the first and last replicates of a treatment increased as the experiment progressed, increasing from one week in treatments A and B to two weeks in treatment $\mathrm{C}$ and to 11 weeks in D. Residues decline logarithmically (to $50 \%$ after one half-life, to $25 \%$ after double this time and $12.5 \%$ after three half-lives). Therefore the slower the residue decreases (i.e. the shallower the curve), the longer the time interval representing any measure of variation between replicates around the mean residue.

The standard and UV opaque films extended the effective residual life of cypermethrin for up to six months. Even the standard UV low film extended kill of the beetles to 17 weeks compared with four weeks outside the tunnels. Thus cypermethrin residues on crops in polytunnels persist, with all the potential damage to beneficial insecticides so characteristic of pyrethroids, long after they would have been degraded on crops grown in the field. Even the UV transmitting cladding, designed to be less opaque to UV than the standard plastic, killed T. confusum for eight weeks.

The three weeks of effective residue life outside the tunnels was longer than the literature suggests applies in the open. A half-life on wheat of only four to eight days (Westcott and Reichle, 1987) and a reduction of residue on strawberry foliage to $40 \%$ after one day, $12 \%$ after three days and only $0.5 \%$ after seven days (Bélanger et al., 1990) has been reported. In the latter example, light rain is known to have occurred on day three. In our experiment, it was necessary to protect the hardboards outside the tunnels from rain, 
and although this was done using the thinnest and clearest plastic film available, it still blocked 10-20\% of the UV and this would certainly have extended the life of the residue. However, most of the increased effective residual life of the insecticide outside the tunnels in our experiment will have been due to the much higher concentration of cypermethrin used. In contrast to our $0.04 \%$ a.i., Bélanger et al. used only $0.0012 \%$ a.i. An $88 \%$ reduction after 3 days would have reduced the residues to 0.0048 (still 4 times the starting concentration used by Bélanger et al.) and $0.00014 \%$ respectively. Another factor to consider is that the hardboards were removed from the polytunnels into the dark of an incubator for two days per fortnight (i.e. $8 \%$ of the time) once the station concerned was included in the bioassay.

In practical terms, therefore, our results should not be seen as on an absolute scale of weeks, but rather that the UV transmitting film doubled the effective residual life of cypermethrin in the clear plastic pocket (itself much longer than if exposed in the open as in a field application, the standard film more than quadrupled it, and the UV opaque film extended it more than six-fold. The importance of regarding the results as a relative time scale is further emphasised by the fact that the criterion of mortality chosen, inability to walk, clearly did not mean that the cypermethrin residue did not still have damaging effects once the beetles were rated as "alive". Beetle mobility on treated bandage from the UV opaque treatment was still very slight compared with the untreated even 22 months after the cypermethrin had been applied.

Thus the covering of a protected cultivation structure can greatly slow down the degradation of pesticide. Even horticultural glass appears to block UV to an extent similar to the standard plastic (UV low) film (Table I), suggesting a four-fold extension of effective residual life under either covering, with this increasing to an eight-fold extension under the UV opaque film. The practical implications of this are that the recommended intervals between application and crop harvest for synthetic pyrethroids may be far too 
short in protected cropping and that the danger to beneficial insects (bees or those often released there for biological control) may be much longer than at present realised. With organophosphates, the extension of residue life will also be present but much shorter, yet the danger of residues at harvest would be magnified by the greater toxicity to the consumer of these compounds.

On a positive note, however, our experiments suggest that polythene claddings could be used to impart useful longer persistence to natural pyrethrum to enable organic growers to use this highly ephemeral insecticide more effectively.

We thank Dr Bhupinder Khambay of Rothamsted Research for helpful discussions and our colleague Professor Colin Walker for valuable comments on the manuscript. $\mathrm{Mr}$ Khalid and Miss Elizabeth Wild kindly gave occasional but essential help in assessing the bioassays. We are also very grateful to Dr Thomas Döhring of Imperial College Silwood Park Campus for providing the spectrophotometer measurements reproduced in Table I. 


\section{REFERENCES}

AMANO, S., KATAMI, T. and SHIBAMOTO, T. (2002). Effect of ultraviolet-absorbing vinyl film on organophosphorus insecticides dichlorvos and fenitrothion residues in spinach. Journal of Environmental Science and Health B, 37, 291-296.

BÉLANGER, A., VINCENT C. and DE OLIVIERA, D. (1990). A field study of four insecticides used in strawberry protection. Journal of Environmental Science and Health $B, 25,615-625$.

CASIDA, J.E. (1980). Pyrethrum flowers and pyrethroid insecticides. Environmental Health Perspectives, 34, 189-202.

COLE, L.M., CASIDA, J.E. and RUZO, L.O. (1982). Comparative degradation of the pyrethroids tralomethrin, tralocythrin, deltamethrin and cypermethrin on cotton and bean foliage. Journal of Agricultural and Food Chemistry, 30, 916-920.

DOUKAS, D. (2002). Impact of spectral cladding materials on the behaviour of glasshouse whitefly Trialeurodes vaporariorum and Encarsia formosa, its hymenopteran parasitoid. Proceedings of the British Crop Protection Council Conference, Pests and Diseases, Brighton, November 2002, 2, 773-776.

DOUKAS, D. and PAYNE, C.C. (2007). The use of ultra-violet blocking film in insect pest management in the UK: effect on naturally occurring arthropod pest and natural enemy opoulations in a protected cucumber crop. Annals of Applied Biology, 151, 221231.

JONES, D. A. (1995). Environmental Fate of Cypermethrin. Environmental Monitoring and Pest Management Branch, Department of Pesticide Regulation, Sacramento, CA, USA. 10 pp. 
RAMMELL, C.G. and BENTLEY, G.R. (1990). Photodegradation of flystrike control org.... pesticides in wool. New Zealand Journal of Agricultural Research, 33, 85-87. STEVENSON, J.H. (1973). A photostable pyrethroid. Nature, 246, 169-170.

VAN EMDEN, H.F. and SERVICE, M.W. (2004). Pest and Vector Control. Cambridge University Press, Cambridge, UK. 349 pp.

FAHMY, H.S.M., BARAKAT, A.A. and KANDIL, M.A. (1978). The effect of exposure to UV-rays and temperature on $\mathrm{SH}-1467$, sumicidin and triazophos. Proceedings of the 4th Conference on Pest Control, Cairo, 1978, Part 1, 564-570.

SASAKI, T., HONDA, Y., UMEKAWA, M. and NEMOTO, M. (1985). Control of certain diseases of greenhouse vegetables with ultraviolet-absorbing vinyl film. Plant Disease, 69, 530-533.

SCHECHTER, M.S., GREEN, N. and LAFORGE, F.B. (1949). Constituents of chrysanthemum flowers XIV. Cinerolone and the synthesis of related cyclopentalones. Journal of the American Chemical Society, 71, 3165-3173.

WESTCOTT, N. D. AND REICHLE, R. A. (1987). Persistence of deltamethrin and cypermethrin on wheat and sweet clover. Journal of Environmental Science and Health B, 22, 91-101. 
TABLE I

Per cent transmission characteristics of films used in the experiments, as well as of horticultural glass

\begin{tabular}{lccc}
\hline Film & $\begin{array}{c}280-320 \mathrm{~nm} \\
\text { (part of UVB) }\end{array}$ & $\begin{array}{c}321-400 \mathrm{~nm} \\
\text { (UVA) }\end{array}$ & $401-700 \mathrm{~nm}$ \\
\hline A (clear A4 punched pocket) & 86.4 & 82.0 & 91.1 \\
B (UV transmitting) & 68.9 & 82.8 & 85.5 \\
C (standard plastic - UV low) & 14.3 & 51.8 & 85.1 \\
D (UV opaque) & 0.8 & 3.1 & 83.0 \\
Horticultural glass & 16.4 & 56.6 & 83.1 \\
\hline
\end{tabular}

Data are means of three readings from different areas of the material. Those for film A and glass are spectrophotometer readings and the remaining data are spectroradiometer results. 


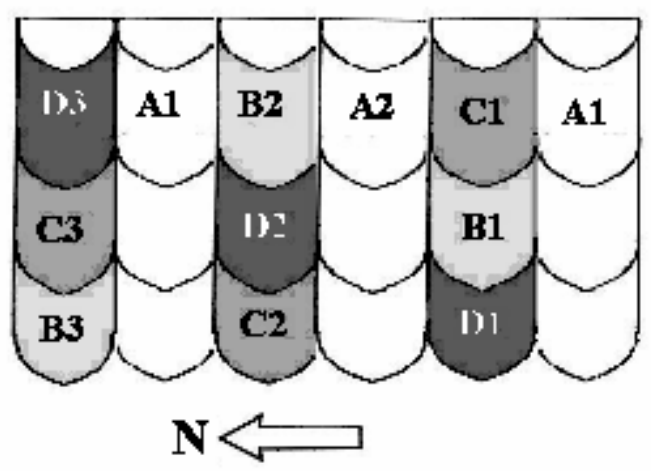

FIG.1

The allocation of cladding treatments to the polytunnel sections and the open unclad areas between the tunnels (not to scale). 1-3, replicates; A-D, treatments of increasing UVblocking, where A = clear A4 envelope outside tunnels, $\mathrm{B}=\mathrm{UV}$ transmitting film, $\mathrm{C}=$ standard UV low film, D = UV opaque film. The shading is for the identification of treatments in the Figure; there was no visual difference between the light passing through claddings B-D.

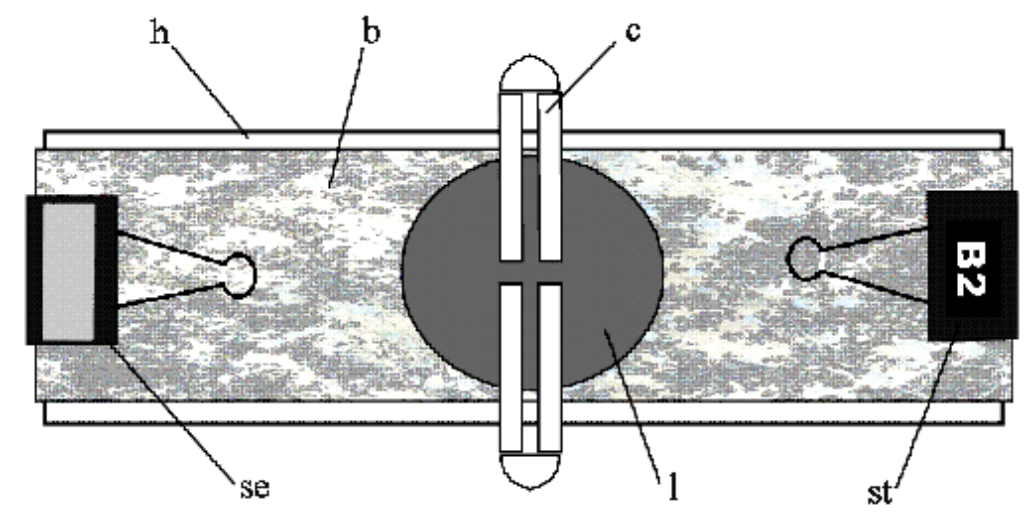

FIG. 2

The bioassay (see text). b, bandage strip; c, bent hair-curl clip; h, hardboard; 1, plastic lid enclosing the beetles; se, small bulldog clip with red, blue or yellow strip; st, small bulldog clip with 'station' identifier'.

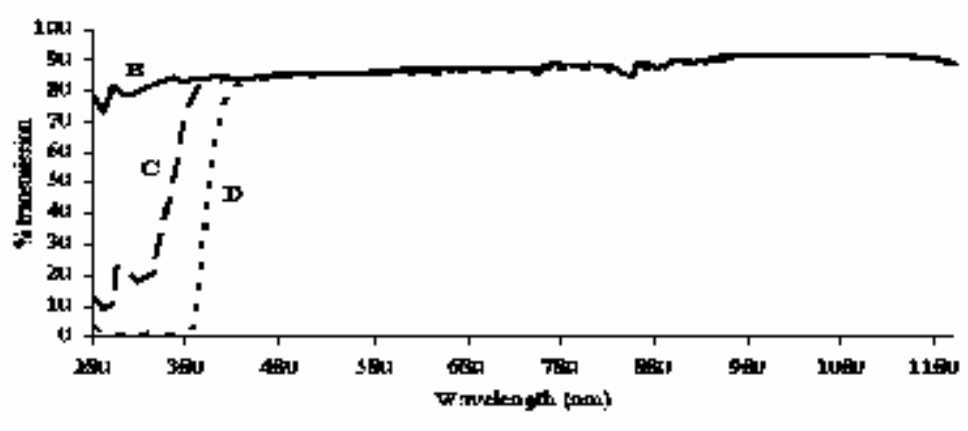


FIG. 3

Per cent transmission spectrum of $\mathrm{B}=\mathrm{UV}$ transmitting film, $\mathrm{C}=$ standard UV low film,

$\mathrm{D}$

$$
=\mathrm{UV}
$$

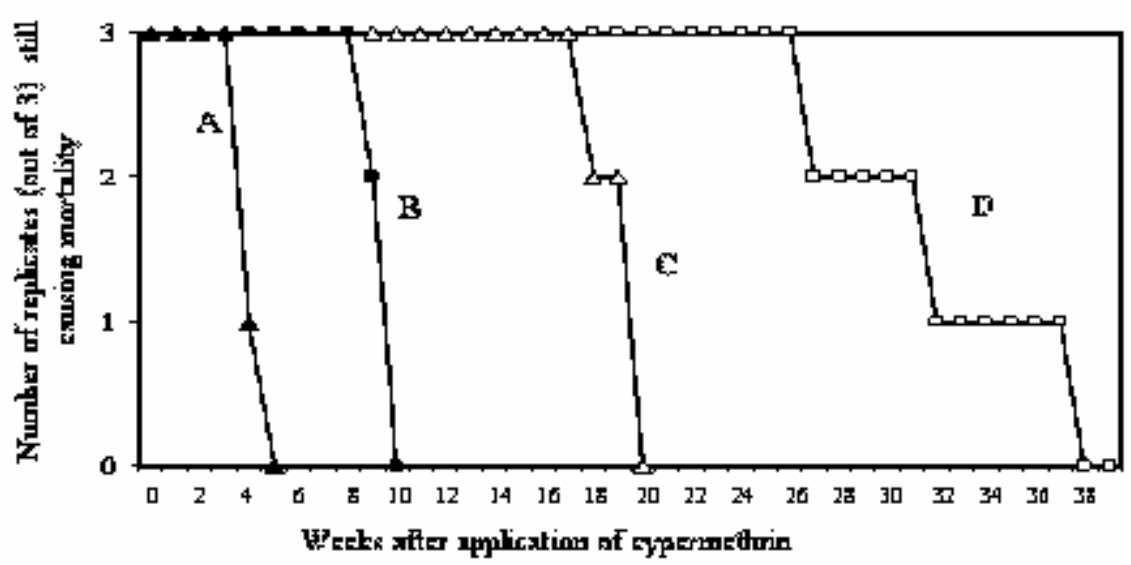

opaque film.

FIG. 4

The number of replicates (out of 3) as the experiment progressed in which the cypermethrin residue has declined to the level that allowed Tribolium confusum adults to survive (48 h bioassay). A = clear A4 envelope, $\mathrm{B}=\mathrm{UV}$ transmitting film, $\mathrm{C}=$ standard UV low film, $\mathrm{D}=\mathrm{UV}$ opaque film.

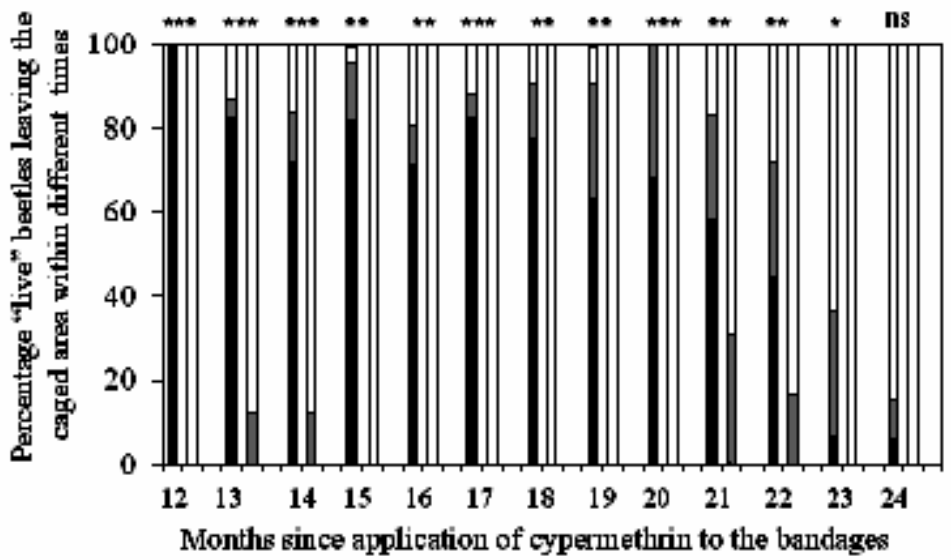

FIG. 5

Percent living beetles in the UV opaque treatment (calculated from the monthly totals) remaining for more than 2 min (black columns) in the caged area on the bandage under a 
lamp once the cage had been removed, those remaining for 21-119 sec (grey columns) and those leaving within $20 \mathrm{sec}$ (white columns. In each pair of columns, the left hand one gives the data for cypermethrin-treated bandage, and the right hand column those for the untreated one. Significance of difference between paired columns ( $\chi^{2}$ calculated from actual frequencies: ***, $P<0.001 ; * *, P<0.01 ; *, P<0.05$; ns, not significant). 